\title{
Simulative Analysis of Influence of Modulation Techniques on COFDM based Radio over Fiber Transmission
}

\author{
Shikha Mahajan \\ Department of Electronics \& Communication Engineering, Panjab University, Chandigarh, India \\ Email: shikhashikha2486@gmail.com \\ Naresh Kumar \\ Department of Electronics \& Communication Engineering, Panjab University, Chandigarh, India \\ Email: naresh_uiet@pu.ac.in
}

\begin{abstract}
In this paper, Coded Orthogonal Frequency Division Multiplexing (COFDM) based Radio over Fiber (RoF) system is simulated (for different bit rates) for different modulation techniques to observe impact on system parameter [quality factor (Q)]. Influence of increasing bit rates has been observed for $2 \mathrm{~km}$ long linear multimode fiber. To reduce dispersion due to multimode fiber, convolutional encoder (code rate $1 / 2$, generator polynomial $133_{8}, 171_{8}$ ) and viterbi hard decision decoding algorithm are chosen for simulative model of COFDM based RoF system. Transmission of 32 sub-carriers with externally modulated continuous wave laser source gave satisfied results for Q values.
\end{abstract}

Index Terms - RoF, COFDM, MMF, Q-factor

\section{INTRODUCTION}

RoF technology is a strong potential candidate for distribution of RF and cellular signals for broadband wireless applications and it takes advantages of intrinsic low attenuation and wide bandwidth for transporting RF signals [1]. The applications of RoF technology include cellular networks, satellite communication, Multipoint Video Distribution Services, Mobile Broadband System and Wireless LANs over optical networks [2]. RF signal/power is carried by a strong optical carrier from central station using optical fiber cables in RoF systems. $\mathrm{RF}$ signal is radiated by using very high gain antenna unit to remote sites [3]. It is used to transmit an analog intensity modulation (IM) of laser diode and to receive direct detection (DD) performed by a photodiode. It is called IM-DD based RoF system, a very simple and low cost RoF communication system. RoF technology involves the use of optical components and techniques to allocate RF signals from the control stations (CS) to the base stations. Thus, RoF makes it possible to centralize the RF signal processing functions in one shared location (CS) [4]. Particularly, radio over multi mode fiber system (RoMMF) has gained much attention recently for its suitability to deliver high-purity signals in analog and digital format for short-reach coverage such as wireless local area networks and ultrawideband radio signals [5]. It is illustrated that although there were recent studies on RoF performance in single mode fibers [6], multi mode fibers can also be allocated for RoF systems with reasonable fiber distances [7]. Multi Mode Fibers (MMFs) were the first optical fibers to be developed in the early days of optical communications, before the advent of Single-Mode Fibers [8].

COFDM has been specified for digital broadcasting systems, Digital Audio Broadcasting, Digital Video Broadcasting [9]. COFDM is particularly well matched to these applications, since it is very tolerant of the effects of multipath (provided a suitable guard interval is used). For short-distance applications, COFDM can be used efficiently for high data rate signal delivery and to tolerate the effect of modal dispersion. Modal dispersion is the dominant performance limiting factor in MMFs. Hence this leads to the need of incorporating COFDM techniques into radio over multi mode fiber systems [9, 10]. In RoF, COFDM impact has been demonstrated with MMF as a promising modulation scheme for mitigating the modal dispersion penalty [11]. Convolutional coding is being used commonly in COFDM systems, and it can give coding gain at different coding rates [12]. By implementing coding and interleaving across sub-carriers, the effect of multipath fading is minimised since interleaving reorganises the number of bits in a way so as to avoid the effects of fading.

In present paper, unidirectional transmission of COFDM based RoF system has been successfully realized by simulations over $2 \mathrm{~km}$ optical distance with convolutional encoder and viterbi hard decision decoder to reduce error floors and increase reliability. The influence of increasing bit rates on performance of Quadrature Amplitude Modulation (QAM) and Quadrature Phase Shift Keying (QPSK) modulation techniques with optical amplifier on received $\mathrm{Q}$ values is studied. 


\section{A. Simulation Setup for Unidirectional RoF System}

RoF system is very cost effective because of localization of signal processing in central station and use of a simple base station. It realizes transparent transformation between RF signal and optical signal.

Pseudo-random bit sequence generator (PRBSG) data format is used and convolutionally encoded at rate $1 / 2$ and generator polynomials $133_{8}$ and $171_{8}$. For downlink simulation, a narrow bandwidth continuous wave $(\mathrm{CW})$ (wavelength $1300 \mathrm{~nm}$ ) from laser diode is modulated via a Mach-Zehnder Modulator (MZM). An ideal pre amplifier before optoelectronic conversion by PINphotodetector having centre frequency of $1300 \mathrm{~nm}$ and post amplifier is used at uplink simulation. Optical link i.e. a linear multi mode fiber of length $2 \mathrm{~km}$ is used. In receiver section, optical signal is detected by a PINphotodiode. Q-factor values of signals are measured by BER analyzer at base station. Fig 1 shows the implementation approach for transmitting convolutionally encoded data over a multi mode fiber and receiving the decoded data.

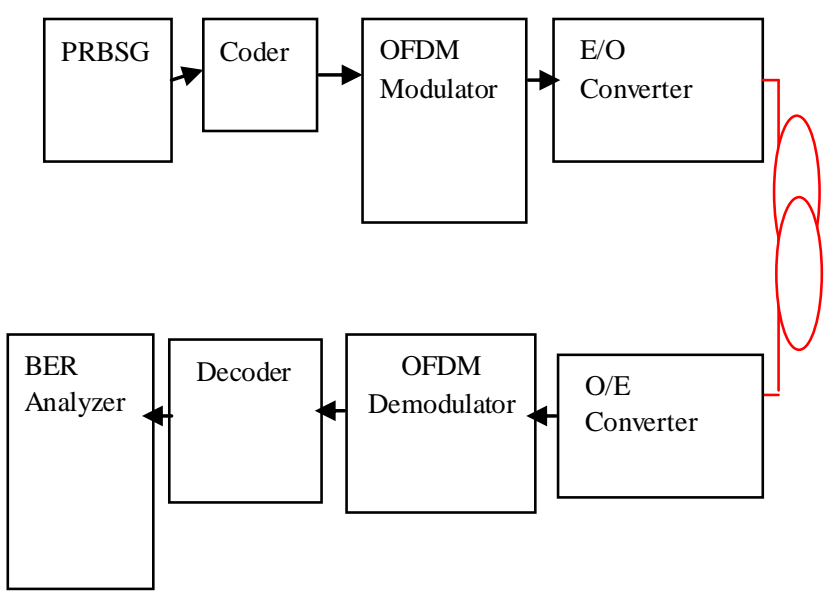

Fig1. Block diagram of COFDM based RoF system.

\section{B. Overview of COFDM System}

The basic principle of COFDM is to split a high rate data stream into a number of lower rate streams to be transmitted simultaneously over a number of subcarriers each of a different frequency and these carriers are orthogonal to each other [13]. The COFDM system consists of three main elements. These are Channel coding/interleaving, IFFT/FFT and Guard interval/cyclic prefix.

Frequency Division Multiplexing (FDM) divides the channel bandwidth into sub channels and transmits multiple low rate signals by carrying each signal on a separate carrier frequency. To ensure that the signal of one sub channel does not overlap with the signal from an adjacent one, some guard band is necessary which is an obvious loss of spectrum and hence bandwidth. But since carriers are orthogonal to each other in COFDM therefore it offers bandwidth efficiency as no guard band is required as shown in Fig 2.
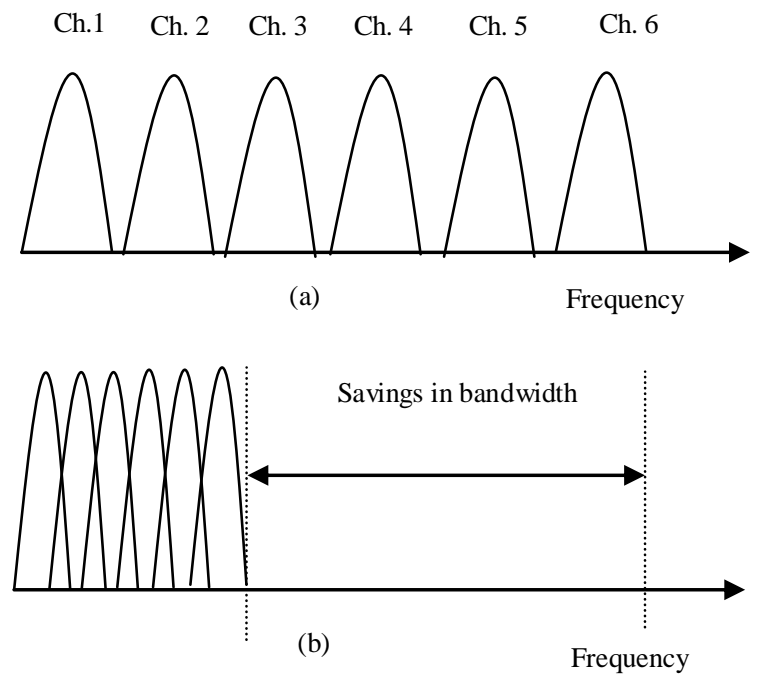

Fig2. Bandwidth conservation (a) FDM (b) OFDM

Orthogonality between sub-carriers is maintained if sinusoids have integer number of cycles in $T_{\mathrm{s}}$ (symbol duration) given by (1) below:

$\int_{0}^{T_{s}} \sin \left(2 \pi f_{c} t\right) \sin \left(2 \pi 2 f_{c} t\right) d t=0$

Where $f_{c}$ is the sub-carrier frequency. The incoming data is first converted from serial to parallel and grouped into x bits each to be modulated by Quadrature Amplitude Modulation (QAM), Quadrature Phase Shift Keying (QPSK), or Binary Phase Shift Keying (BPSK). The required spectrum is then converted back to its time domain signal using an Inverse Fast Fourier Transform (IFFT), commonly used in most applications. The IFFT performs the transformation very efficiently and provides a simple way of ensuring the carrier signals produced are orthogonal. The signals are then converted back to serial for transmission. The discrete signals are converted back to analog. The receiver performs the inverse process of the transmitter.

A guard interval is inserted between every COFDM symbol to avoid Inter symbol Interference (ISI) caused by multipath distortion. This means that in the guard time, the COFDM symbol is cyclically extended to avoid Inter Carrier Interference (ICI) [14]. The guard interval is formed by a cyclic continuation of the signal so the information in the guard interval is actually present in the COFDM symbol [15]. It is actually added by taking the copy of the last portion of the COFDM symbol and placing it at the start of the symbol as illustrated in Fig 3. 


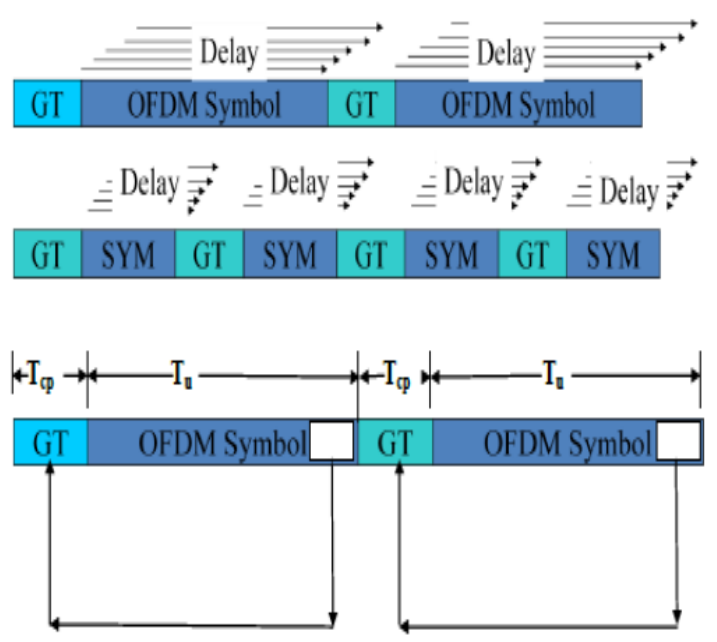

Fig3. Guard time and Cyclic prefix.

The last $\mathrm{T}_{\mathrm{g}}$ portion of the symbol is appended and transmitted during the guard time. $\mathrm{T}_{\mathrm{u}}$ is the COFDM symbol time without guard interval. $\mathrm{T}_{\mathrm{cp}}$ is the duration of the copied information in the guard interval using cyclic prefix. Therefore total COFDM symbol time $\mathrm{T}_{\mathrm{s}}=$ $\mathrm{T}_{\mathrm{u}}+\mathrm{T}_{\mathrm{cp}}$. Cyclic prefix helps in maintaining orthogonality between sub-carriers by converting linear convolution into circular convolution in multipath environment and also avoids ICI.

The Fast Fourier Transform is a very efficient mathematical method for calculating Discrete Fourier Transform (DFT) [15]. The direct evaluation of an Npoint DFT is done using the following formula (2):

$X(k)=\sum_{n=0}^{N-1} x(n) \cdot e^{-\frac{\sqrt{2} 2 \pi n k}{N}}$

Where $\mathrm{k}=0,1,2 \ldots \ldots \mathrm{N}-1$. DFT requires $\mathrm{N}^{2}$ complex multiplications and $\mathrm{N}^{*}(\mathrm{~N}-1)$ complex additions whereas use of FFT algorithm reduces the number of computations to the order of N/2*log2 (N) complex multiplications and $\mathrm{N}^{*} \log _{2}(\mathrm{~N})$ additions. Moreover FFT algorithm works efficiently when $\mathrm{N}$ is a power of 2, therefore the number of sub-carriers is usually kept as power of 2 . IFFT/FFT operation ensures that sub-carriers do not interfere with each other. IFFT is used at the transmitter to obtain the time domain samples of the multicarrier signal. FFT is used to retrieve the data sent on individual sub-carriers. Therefore COFDM has a very simple implementation capability.

COFDM system employs conventional forward error correction codes and interleaving for protection against burst errors caused by deep fades in the channel. Often it is concatenated with a block code for improving the performance. A Viterbi decoder for decoding a convolutional code is easy to implement. Codes of different coding rates are used in conjunction with different modulation schemes to support different quality of services. While error correction codes provide coding gain in the system, interleaving provides diversity gain. For OFDM, when a deep fade occurs in the channel, the bits within the deep fade are erased. Interleaving the bits across different frequency bins distributes the energy within a symbol among different sub-carriers. Since distinct sub-carriers undergo different fading conditions, the probability that all the bits corresponding to a symbol are lost, decreases significantly. An uncoded OFDM system cannot exploit frequency diversity, since in frequency selective fading channels, the sub-carriers separated by coherence bandwidth are independent of each other. The frequency diversity can be realized by spreading coded bits over different sub-carriers in such a way that adjacent bits are separated by coherence bandwidth. An interleaver permutes symbols according to a mapping. A corresponding deinterleaver uses the inverse mapping to restore the original sequence of symbols. Interleaving and deinterleaving can be useful for reducing errors caused by burst errors in a communication system. A convolutional interleaver consists of a set of shift registers, each with a fixed delay. Fig 4 shows a convolutional encoder with constraint length $\mathrm{L}=7$.

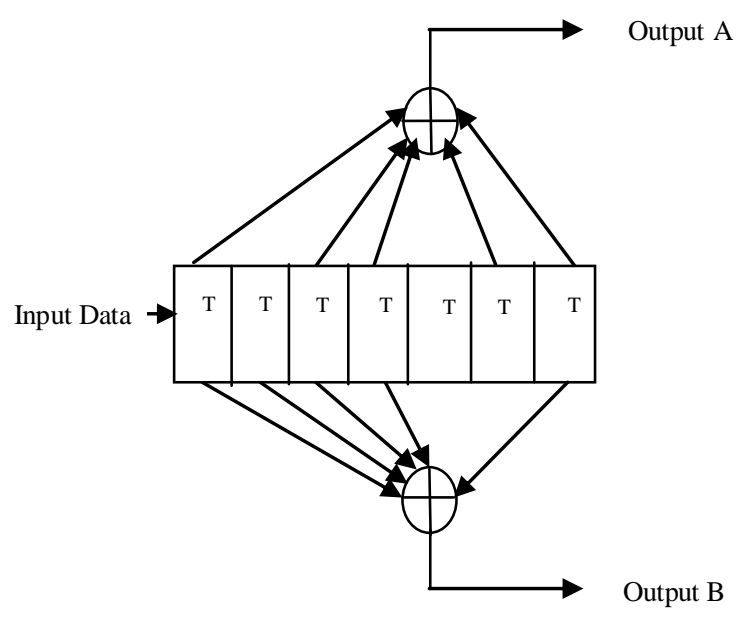

Fig4. Convolutional encoder.

\section{Viterbi Algorithm for Decoding Convolutional Codes}

Viterbi algorithm is a well-known maximumlikelihood algorithm for decoding of convolutional codes. A hard decision decoder receives only bits from the channel (without any reliability estimate). A Viterbi algorithm consists of the following parts:

1. Branch metric calculation - calculation of a distance between the input pair of bits and the four possible "ideal" pairs ("00", "01", "10", "11”). For a hard decision decoder it is a Hamming distance, i.e. a number of differing bits.

2. Path metric calculation - for every encoder state, calculate a metric for the survivor path ending in this state (a survivor path is a path with the minimum metric). 


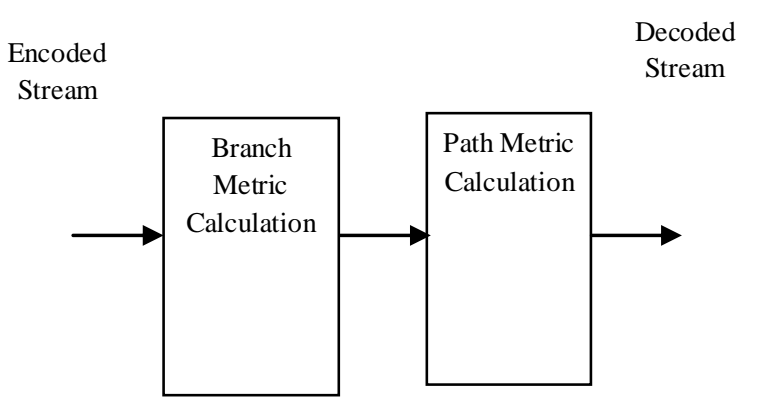

Fig5. Parts of viterbi algorithm

\section{COFDM Model}

Convolutional Encoder: According to the COFDM standard, information data must be encoded with a convolutional encoder with coding rate $\mathrm{R}=1 / 2,2 / 3$, or $3 / 4$, corresponding to the desired data rate. The convolutional encoder uses the industry-standard generator polynomials, $\left[133_{8}, 171_{8}\right]$ of rate $\mathrm{R}=1 / 2$ [16].

FFT and IFFT: This sub-block is the heart of COFDM technology. These operations performing linear mappings between $\mathrm{N}$ complex data symbols and $\mathrm{N}$ complex COFDM symbols result in robustness against fading multipath channel. The reason is to transform the high data rate stream into $\mathrm{N}$ low data rate streams, each experiencing a flat fading during the transmission. Length of FFT/IFFT is taken to be 64 .

Guard Time Insertion: This sub-block is responsible for making COFDM symbols resistant to inter symbol interference. It takes copy of last samples equal to guard interval from each COFDM symbol and place it at the beginning of the COFDM symbol. The guard period used is symbol extension in COFDM model.

Viterbi Decoding: For decoding the convolutional codes the Viterbi decoding algorithm [17] is used, which offers best performance according to the maximum likelihood criteria. The input to the Viterbi decoder is hard-decided bits that are 0 or 1 , which is referred to as a hard decision. The implementation process of COFDM is as follows:

1) The data bits are convolutionally encoded with a code rate $1 / 2$.

2) The encoded bits are bit-interleaved and then mapped into in-phase and quadrature components of the complex symbol.

3) The complex symbols are modulated by OFDM modulator. Table 1 summarises COFDM parameters.
Table 1.COFDM parameters

\begin{tabular}{ll}
\hline Parameter & Value \\
\hline Code rate & $1 / 2$ \\
Generator polynomials & $133_{8}, 171_{8}$ \\
Constraint length & 7
\end{tabular}

The implementation process of Viterbi algorithm to convolutionally decode binary data is as follows:

1) Decoded $=$ Vitdec(Code,Trellis, Tblen,Opmode,D ectype)

2) The vector Code was decoded using the Viterbi algorithm. Code was assumed to be the output of a convolutional encoder specified by the MATLAB $^{(\mathrm{R})}$ structure Trellis.

3) Poly2trellis was used for a valid Trellis structure. Each symbol in Code consists of $\log _{2}$ (Trellis.numOutputSymbols) bits.

4) Decoded is a vector in the same orientation as Code and each of its symbols consists of $\log _{2}$ (TRELLIS.numInputSymbols) bits.

5) Tblen is a positive integer scalar that specifies the traceback depth. Table 2 summarises the Viterbi algorithm parameters.

Table 2.Viterbi algorithm parameters

\begin{tabular}{ll}
\hline Parameter & Value \\
\hline numInputSymbols & 2 \\
numOutputSymbols & 4 \\
numStates & 128 \\
tblen & 7 \\
opmode & trunc \\
dectype & hard \\
& \\
\hline
\end{tabular}

\section{E. MMF Response Model}

The block diagram of the ROMMF system used for analysis and simulation is shown in Fig 6. In this system the COFDM signal generated out of the OFDM transmitter was directly injected into the laser diode (LD) at the desired signal level. The optical intensity signal out of the LD is fed into an MMF followed by a photodetector. The received COFDM signal will be demodulated and decoded to retrieve the data. This system can be used for analysing the performance of the optical link alone for the OFDM input. Table 3 summarizes MMF parameters. 


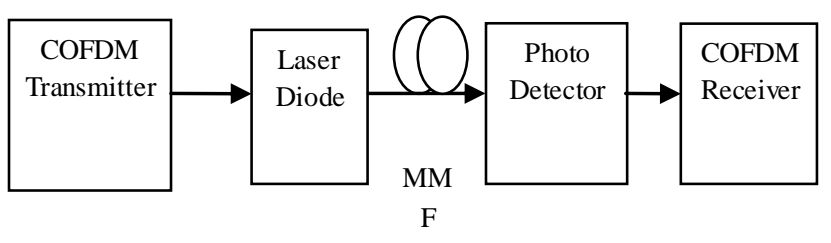

Fig6. Demonstration of ROMMF system under intensity modulation and direct detection.

Table 3.MMF parameters

\begin{tabular}{ll}
\hline Parameter & Value \\
\hline Fiber dispersion & \\
& $-100 \mathrm{ps} /(\mathrm{nm}$ \\
$\mathrm{km})$ & $1.25 \mathrm{~dB} / \mathrm{km}$ \\
Fiber attenuation & $1300 \mathrm{~nm}$ \\
Wavelength & $2 \mathrm{~km}$ \\
Fiber length & \\
\hline
\end{tabular}

\section{RESULTS AND DISCUSSION}

Up to this moment, the simulation only considers some important combination of parameters that are dominant in optical data transmission. The encoded sequence is split into 32 sub carriers. The data is then modulated using $16 \mathrm{QAM} / 4$ bits per symbol or QPSK/ 4 bits per symbol, then carried by different frequencies of each sub carrier to have the orthogonality between the sub carriers. All other parameters that have been used in this transmitter part are already explained in the previous section. Fig 7 and 8 shows the RF spectrum for I (in phase) and Q (quadrature) component of OFDM modulator.

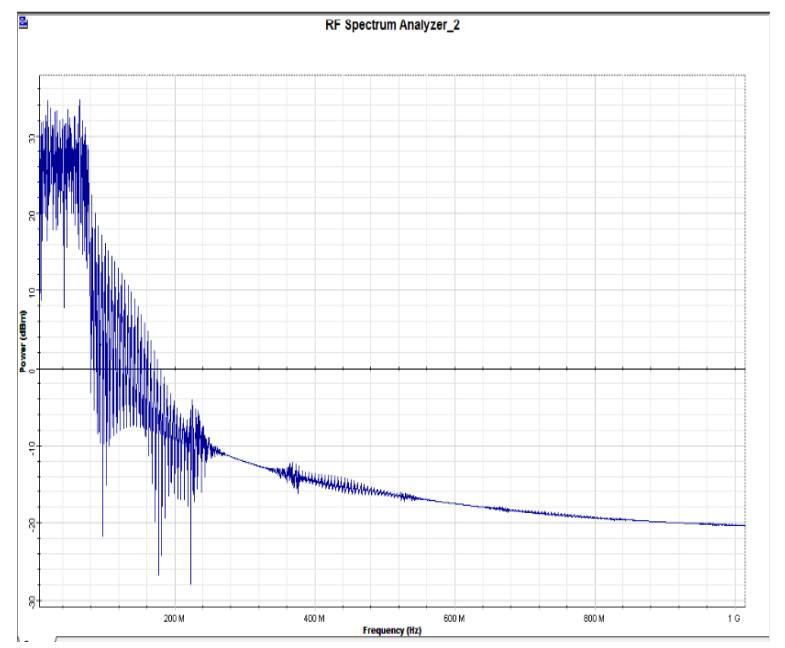

Fig7. RF spectrum of I component of OFDM modulator.

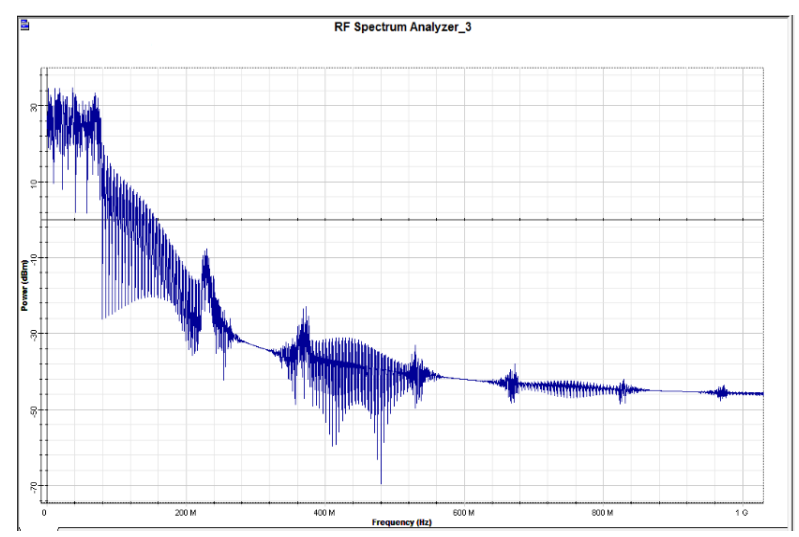

Fig8. RF spectrum of Q component of OFDM modulator.

Further in this section results from the transmission link, which are in optical domain, are presented. The function of transmission link is to convert the electrical domain signal from the transmitter part into optical domain signal so the COFDM signal could be sent through the fiber. To do this CW laser and external modulator i.e. MZM to modulate the signal of COFDM is used. The wavelength for CW laser is set to $1300 \mathrm{~nm}$, while the rest are to be set into default values from the Optisystem. The fiber length for the transmitting the signals is $2 \mathrm{Km}$. The result from transmission link after COFDM signal was optically modulated by MZM is shown in Fig 9 where the power of the signal is 69.0592dBm.

\section{롤 Optical Spectrum Analyzer}

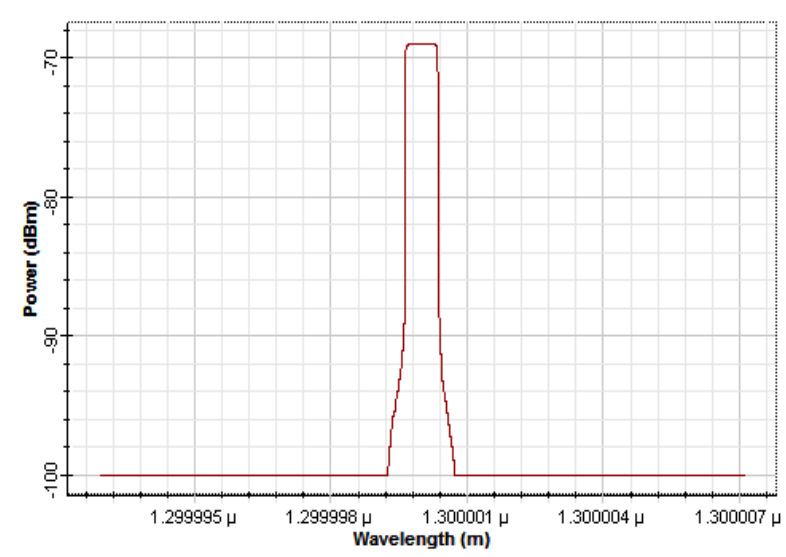

Fig9. COFDM optically modulated by MZM

After optically modulated, the signal is then transmitted through the pre-amplifier and then fiber link. The signal after MMF can be seen from the Fig 10 below. 


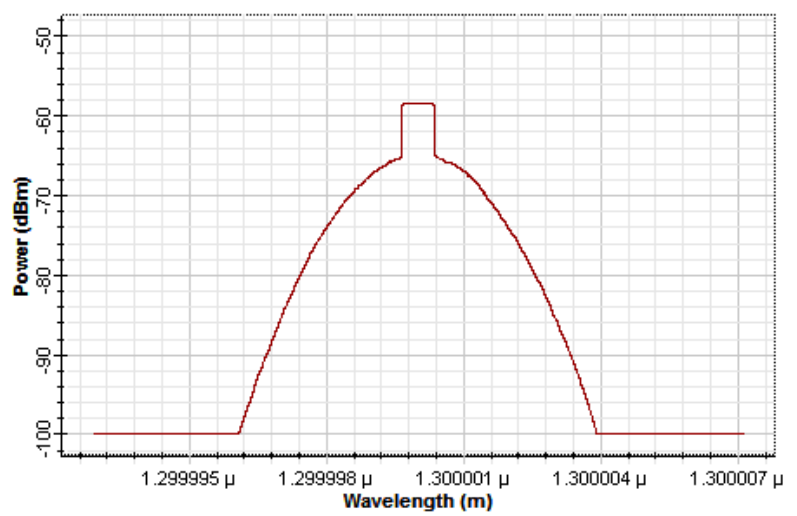

Fig10. Signal through fiber.

From both Fig 9 and 10 it can be seen that the wavelength is $1300 \mathrm{~nm}$ but the power from both signals is different. The optically modulated signal is amplified (gain $12 \mathrm{~dB}$ ) and passed through MMF. From the Fig 10 above it can be analyzed that now signal has increased power from $-69.0592 \mathrm{dBm}$ to $-58.5374 \mathrm{dBm}$. The amplification is necessary to be done before the signal is transmitted through the optical fiber to reduce the effect of loss possibility from the signal. Further the optical signal from MMF is post-amplified and is shown in the Fig 11. The signal power further increased from $58.5374 \mathrm{dBm}$ to $-45.3038 \mathrm{dBm}$.

ㅂ

Optical Spectrum Analyzer_2

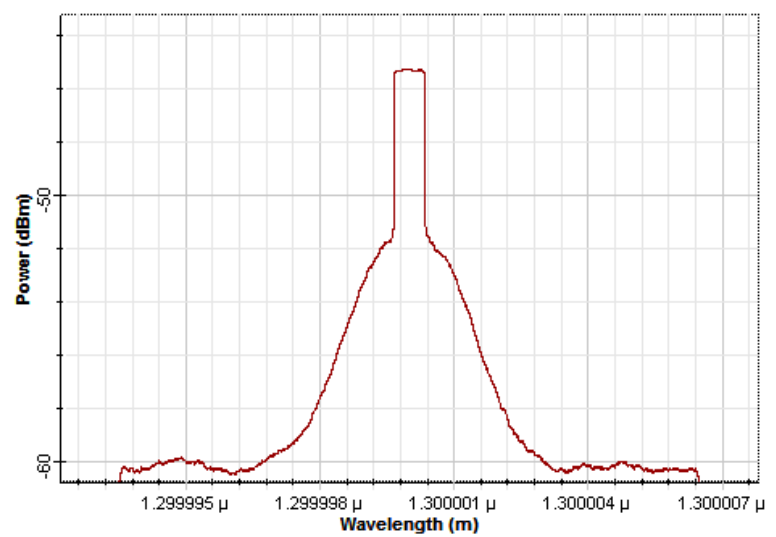

Fig11. Signal after post-amplification.

After optically modulated and being transmitted over fiber link, the optical domain signal is then converted back into electrical domain signal by PIN photodetector at the receiver part. The PIN photodetector used in this simulation has responsivity $1 \mathrm{~A} / \mathrm{W}$. Fig 12 and 13 below show the I and Q components of OFDM demodulator using M-ary Sequence Visualizer.
M-ary Sequence Visualizer

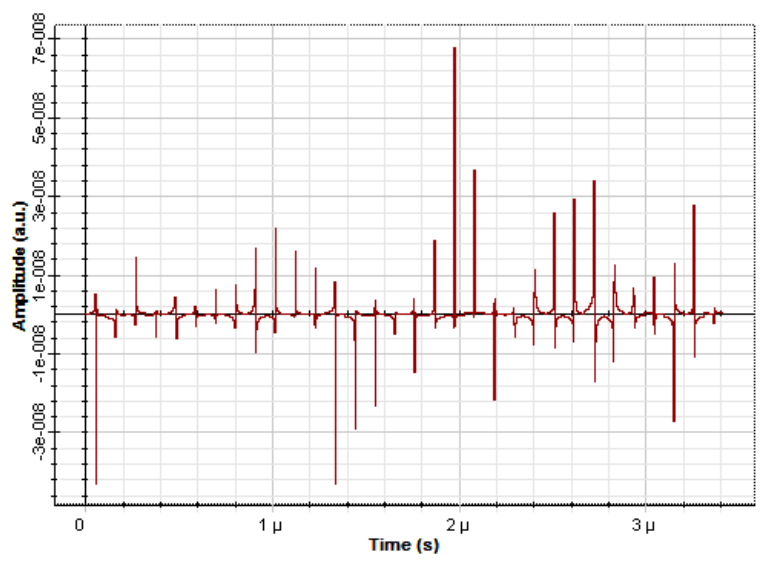

Fig12. M-ary sequence of I component of OFDM demodulator.

붑

M-ary Sequence Visualizer_1

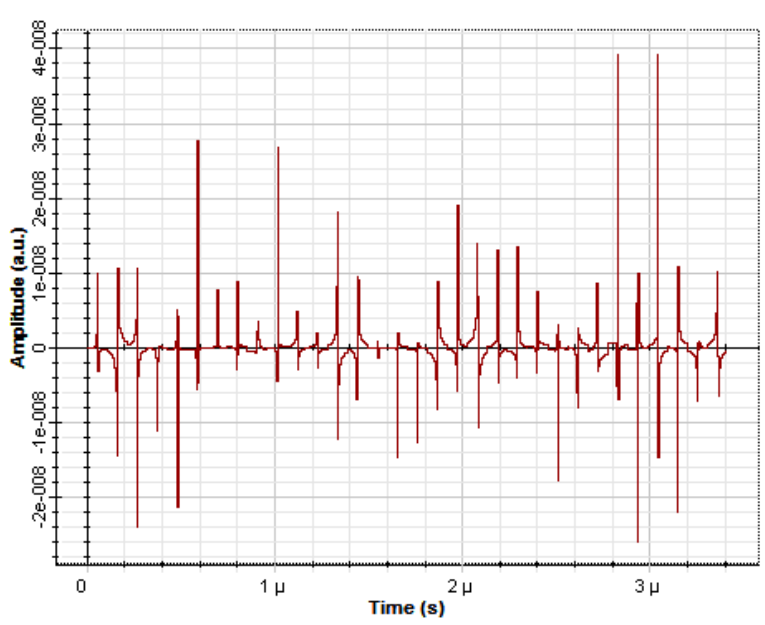

Fig13. M-ary sequence of Q component of OFDM demodulator.

BER analyzer, both a visualizer and a receiver component is used, in that it simulates the conversion of electrical pulses into logical $1 \mathrm{~s}$ and $0 \mathrm{~s}$ and gives $\mathrm{Q}$ factor values. Firstly the simulative model's performance was analyzed for COFDM/OFDM for radio over multi mode fiber system. The Fig 14 below shows the analysis of COFDM and OFDM with 16 QAM modulation technique based upon the same set of parameters for both the techniques. It can be seen from the Fig below that QAM when used with coded OFDM gives higher values of $\mathrm{Q}$ than used with uncoded OFDM, such as at $600 \mathrm{Mbps}$ COFDM (QAM) gives $\mathrm{Q}=8.214$ while OFDM (QAM) gives $\mathrm{Q}=7.447$ respectively. Also as the data rate increases the $\mathrm{Q}$ factor degrades below the valid $Q$ value $(Q=6$ for telecommunication systems) for both COFDM and OFDM. Typical error rates for optical fiber telecommunication systems range from $10^{-9}$ to $10^{-12}$. It is quoted that BER value $10^{-9}$ corresponds to a $\mathrm{Q}$ value 6 [18]. 


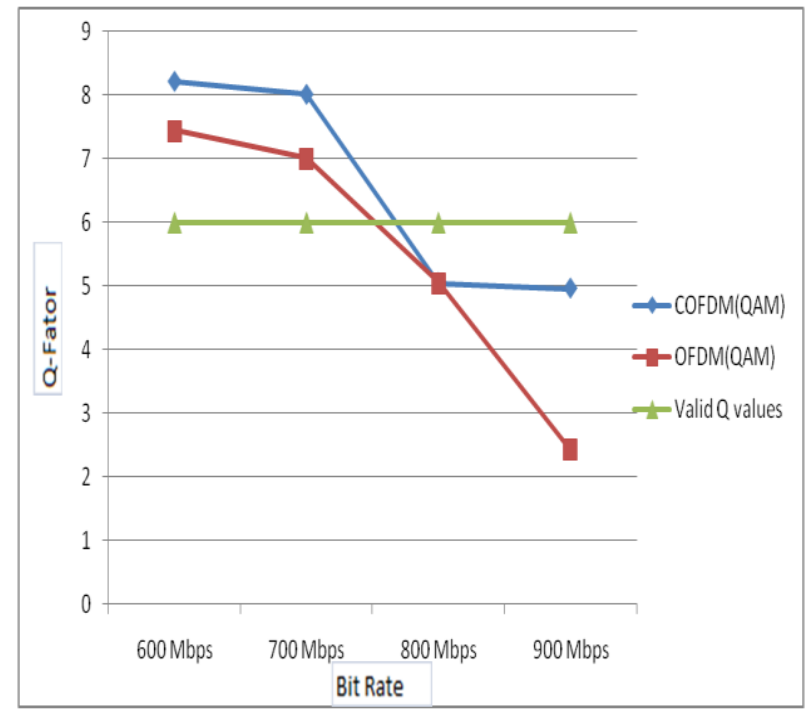

Fig14. Analysis of COFDM and OFDM with 16 QAM.

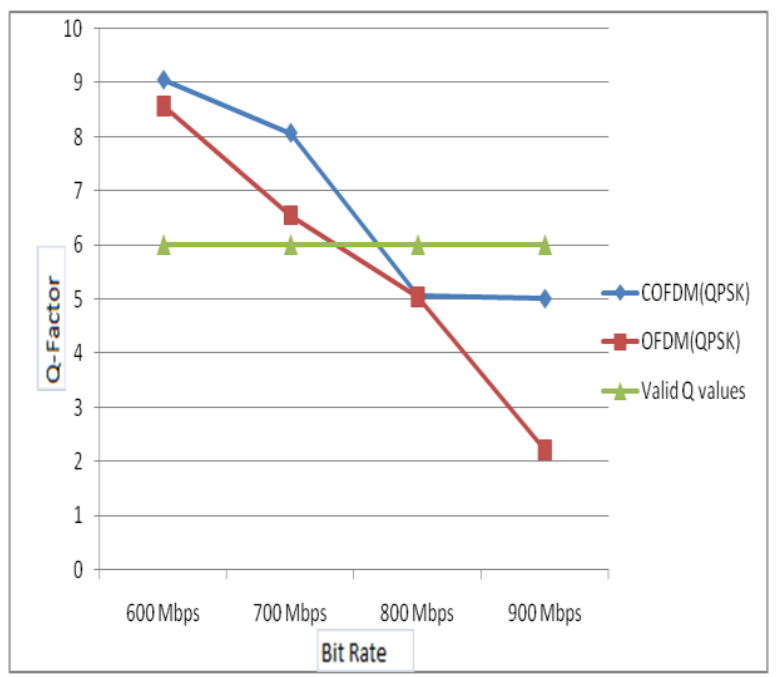

Fig15. Analysis of COFDM and OFDM with QPSK.

Then the model was analyzed for its performance with QPSK modulation technique for both COFDM and OFDM. The Fig 15 above shows the analysis of COFDM and OFDM with QPSK modulation technique based upon the same set of parameters. It can be seen from the Fig that QPSK when used with coded OFDM gives higher values of $Q$ than used with uncoded OFDM, such as at $600 \mathrm{Mbps}$ COFDM (QPSK) gives $\mathrm{Q}=9.050$ while OFDM (QPSK) gives $\mathrm{Q}=8.572$ and at $700 \mathrm{Mbps}$ COFDM (QPSK) gives $\mathrm{Q}=8.070$ while OFDM (QPSK) gives $\mathrm{Q}=6.550$ respectively. Further as the data rate increases the $\mathrm{Q}$ factor degrades below the valid $\mathrm{Q}$ value $(\mathrm{Q}=6$ for telecommunication systems) for both COFDM and OFDM. From both the Fig's 14 and 15 COFDM gave higher values of Q-Factor than OFDM for same set of simulative parameters.
From [19] Optical Amplifier gives higher values of Q-Factor than EDFA with either of the modulation techniques (QAM/QPSK). So the performance of the simulative model (COFDM based RoMMF System) was analyzed to find the better modulation technique between QAM and QPSK when used with Optical Amplifier in terms of parameter Q-factor, for same set of simulative parameters.

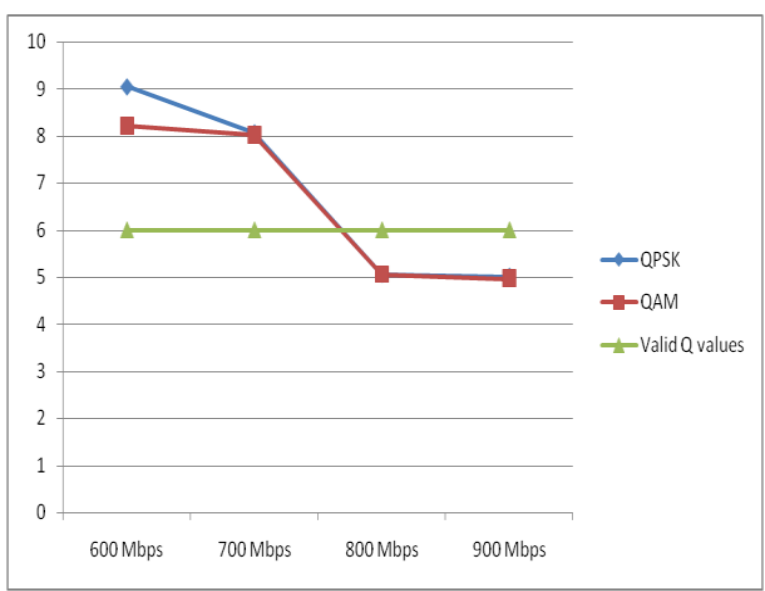

Fig16. Analysis of QAM/QPSK using optical amplifier

Here Fig 16 above shows the performance analysis for QAM/QPSK when used with an Optical Amplifier in COFDM based RoMMF system. It can be seen that for bit rates used in simulation QPSK with Optical Amplifier gives higher Quality Factor than QAM gives with Optical Amplifier. As from the Fig at $600 \mathrm{Mbps}$ $\mathrm{QAM}$ gives $\mathrm{Q}=8.214$ while $\mathrm{QPSK}$ gives $\mathrm{Q}=9.050$. Therefore between QPSK and QAM modulation technique when used with Optical Amplifier for this simulative model for same set of simulative parameters, QPSK can be seen as better modulation technique (in terms of Q).

\section{CONCLUSIONS}

The study of incorporating COFDM modulation technique with RoF technology was essential for nowadays implementation of optical communication. The combination of the advantages from COFDM and RoF provided a medium haul communication network. In this paper, the simulation of COFDM scheme for RoMMF system has been completely done by using software Optisystem and MATLAB ${ }^{(\mathrm{R})}$, for different modulation techniques to observe impact on system parameter [Quality Factor (Q)]. Some consideration has been added to system design in order to meet the applicable design for using in the reality. Influence of increasing bit rates has been observed for $2 \mathrm{~km}$ long linear multimode fiber. To reduce loss due to multi mode fiber, convolutional encoder (code rate 1/2, generator polynomial $133_{8}, 171_{8}$ ) and Viterbi hard decision decoding algorithm are chosen for simulative model of COFDM based RoF system. 
The model was analyzed for COFDM to be better technique than OFDM for RoMMF system. The analysis showed that QAM/QPSK modulation techniques gave higher $\mathrm{Q}$ values (at $600 \mathrm{Mbps}$ COFDM (QAM) gave $\mathrm{Q}=8.214$ while OFDM (QAM) gave $\mathrm{Q}=$ 7.447 respectively and at $600 \mathrm{Mbps}$ COFDM (QPSK) gave $\mathrm{Q}=9.050$ while $\mathrm{OFDM}(\mathrm{QPSK})$ gave $\mathrm{Q}=8.572$ and at $700 \mathrm{Mbps}$ COFDM (QPSK) gave $\mathrm{Q}=8.070$ while OFDM (QPSK) gave $\mathrm{Q}=6.550$ respectively) when used with COFDM rather than with OFDM for same simulative model using same set of parameters (from Fig No. 14 and 15). Since in [19] it was concluded that Optical Amplifier gave higher Q values than EDFA with COFDM based RoMMF system, further analysis using Optical Amplifier was done in this paper to analyse better modulation technique between QAM/QPSK for this simulative model. QPSK modulation technique gave better results (i.e. higher $\mathrm{Q}$ values as at $600 \mathrm{Mbps} \mathrm{QAM}$ gave $\mathrm{Q}=8.214$ while QPSK gave $\mathrm{Q}=9.050)$ than $\mathrm{QAM}$ modulation technique (from Fig No. 16). This system design is best suitable for medium haul communication using MMF with QPSK modulation technique and using optical amplifier for reducing the signal loss.

\section{ACKNOWLEDGMENT}

In the name of almighty I would like to give sincere appreciation to Prof. Renu Vig, Director, University Institute of Engineering and Technology, Panjab University for providing the infrastructure and environment that made everything run smoothly. I am also very grateful to my parents and friends for their worthy co-operation, without their help, it would not have been possible to complete this study.

\section{REFERENCES}

[1] Hamed Al Raweshidy, Shozo Komaki, Radio over fiber technologies for mobile communications networks, pp. 128-132 (2002).

[2] Ng'oma, Design of a radio-over-fiber system for wireless LANs, Report Number: tue wp6 pub 02 v01, (2002).

[3] Guennec, Y.1., Pizzinat, A., Meyer, S., et al., Lowcost transparent radio-over-fiber system for inbuilding distribution of UWB signals, pp. 2649-2657 (2009).

[4] E. Sackinger, Broadband Circuits for Optical Fiber Communication, John Wiley \& Sons, p. 11 (2005).

[5] Stott, J.H., The DVB terrestrial (DVB-T) specification and its implementation in a practical modem, pp. 255-260 (1996).

[6] I. Haroun and F. Gouin, WLANs meet fiber optics: evaluating 802.11a WLANs over fiber optic links, http: //rfdesign.com/ar/radio-wlans-meet-fiber/.

[7] Tolga Kurt, Abbas Yongacoglu and Jean-Yves Chouinard, OFDM and Externally Modulated Multimode Fibers in Radio over Fiber Systems, IEEE Transactions on wireless communications, pp. 26692674 (2006).
[8] D. Gloge and A. J. Marcatili, Multimode theory of graded-core fibers, Bell Syst. Tech. J. 52, p.1563 (1973).

[9] A.M. Matarneh, S.S.A. Obayya, Bit-error ratio performance for radio over multimode fiber system using coded orthogonal frequency division multiplexing, pp. 151-157 (2011).

[10] Aser M. Matarneh, S. S. A. Obayya, I. D. Robertson, Coded orthogonal frequency division multiplexing transmission over graded index multimode fiber, (2007).

[11] Shikha Mahajan and Naresh Kumar, COFDM for Radio over Multimode Fiber: a Review, IJCA special issues on IP Multimedia Communications, pp. 68-71 (2011).

[12] Arun Agarwal, Kabita Agarwal, Design and Simulation of COFDM for High Speed Wireless Communication and Performance Analysis, pp. 22-28 (2011).

[13] Dixon, B.J., R.D., Iezekiel, S., Orthogonal frequency-division multiplexing in wireless communication systems with multimode fiber feeds, pp. 1404-1409 (2001).

[14] Weon-cheol Lee, Hyung-Mo Park, Kyung-jin Kang and Kuen-bae Kim, Performance Analysis of Viterbi Decoder Using Channel State Information in COFDM System, IEEE transactions on broadcasting vol. 44, pp. 488-496 (1988).

[15] Ramjee Prasad, OFDM for wireless Communications systems, Artech House (2004).

[16] William Y. Zou and Yiyan Wu, COFDM: An Overview, IEEE transactions on broadcasting, vol.41, pp 1-8 (1995).

[17] Aníbal Luis Intini, Orthogonal Frequency Division Multiplexing for Wireless Networks, University Of California, Santa Barbara (2000).

[18] Gerd Keiser, Optical Fiber Communications, McGraw-Hill Series in Electrical Engineering, (2000).

[19] Shikha Mahajan, Naresh Kumar, Performance Analysis of Coded OFDM Signal for Radio over Fiber Transmission, IORS Journal of Electrical and Electronics Engineering, Volume 1,pp. 49-52 (2012).

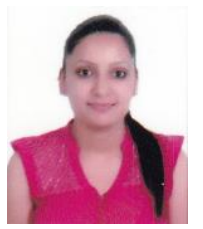

Shikha Mahajan is currently doing M.E from University Institute of Engineering and Technology, Panjab University, Chandigarh. She has received her B.Tech degree from Punjab Technical University, Jalandhar, India. Her interests include Radio over Fibers and Computer Networks.

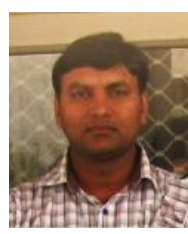

Naresh Kumar received his M.E. Degree in Electronics Engineering from PEC, Chandigarh in 2004. Presently he is working as an Assistant Professor at UIET, Panjab University Chandigarh since 2005. His field of specialization includes Digital \& wireless communication, Cognitive Radio Networks, Radio over fiber etc. 